\title{
Colóquio "A República no Brasil: Idéias e Experiências" - França
}

Nos dias 18, 19 e 20 de janeiro de 1990, ocorreu na França um colóquio sobre "A República no Brasil: Idéias e Experiências", patrocinado pelas entidades: Universidade de Paris-Sorbonne (Paris IV), Instituto de Pesquisas sobre as Civilizaçóes do Ocidente Moderno, Instituto de Estudos Avançados da USP e CNPq.

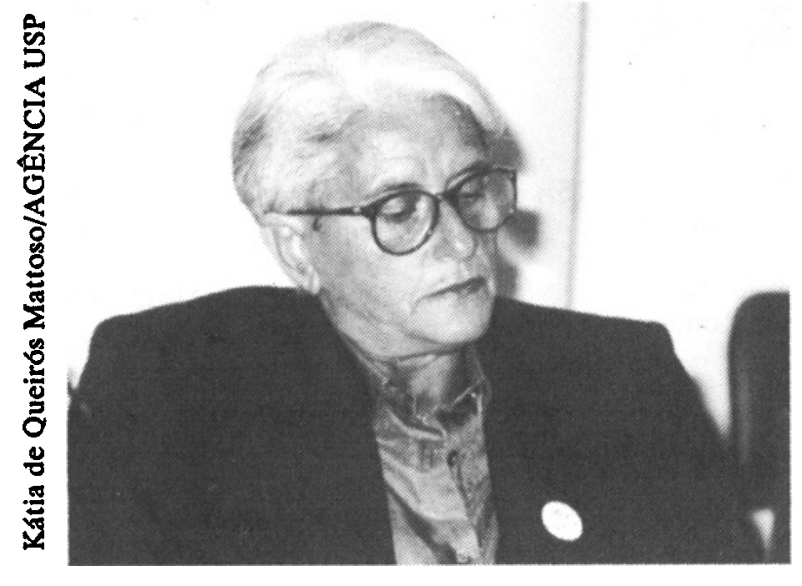

Este colóquio foi coordenado pelos profs. Katia de Queirós Mattoso, François Crouzet, Carlos Guilherme Mota e Ismênia de Lima Martins, e tratou dos seguintes temas:

teóricas e sobre as experiências republicanas que se

1) Idéias e experiências republicanas no mundo ocidental, da Antigüidade Clássica à Época Moderna

Estão agrupados nesse tema os trabalhos históricos sobre as principais formulaçōes tornaram patrimônio cultural comum do Ocidente, seja como referência constante para a formulaçáo ideológica, seja como modelo de açáo política.

2) Idéias e experiências republicanas contemporâneas

Estão agrupados nesse tema os trabalhos sobre as idéias, as instituiçóes políticas $\mathbf{e}$ as experiências históricas dos países que adotaram um governo republicano para forjar seu Estado Nacional. Os Estados Unidos e a França (particularmente, por sua experiência sob a Terceira República), foram escolhidos como paradigmas pelos ideólogos, políticos e militares responsáveis pela implantação da República no Brasil.

3) A República no Brasil

Nesse terceiro tema, estão agrupados os trabalhos sobre a República brasileira, dando uma atençâo particular náo apenas aos aspectos políticos (estruturas jurídicas, partidos políticos, instituiçóes e ideologias), mas também aos aspectos conjunturais da crise política, social e econômica que determinaram a Proclamação da República em 1889. 
MATIN: $9 \mathrm{~h}-12 \mathrm{~h} 45$

Amphi, Louis Liard

9h Enregistrement et accueil des participants

$9 \mathrm{~h} 30$

Ouverture du Colloque

Allocution de Monsient Michel

MESLIN, président de l'

Université de Paris-Sorbonne

(Paris, IV);

Allocution de Madame Katia de QUEIROS MATTOSO, professeur de histoire moderne et contemporaine du Brésil à l'

Université de Paris-Sorbonne

(Paris IV), responsable du

Colloque.

10h Overture des travaux.

Président de séance: $M$. Claude

FOHLEN.

Communications:

République et démocratie dans

le monde gree antique

M. Andr LARONDE, professeur

à l'.Université de Paris-Sorbonne

(Paris, IV)

10h30 Pause. Café.

10h45 L'idée de la "res publica" sous I'Empire Romain M. Jean-Pierre MARTIN, professeur à l' Université de

Paris-Sorbonne

(Paris IV)

IIh15 L'évolution du concept

"République" à l'époque des guerres civiles romaines M. Francois HINARD, professeur à l'Université de Paris-Sorbonne (Paris IV)

$11 \mathrm{~h} 45$ Discussion.

$12 \mathrm{~h} 45$ Déjeuner.

APRES-MIDI: $14 \mathrm{h30}$ - i7h45

Amphi, Louis Liard

Président de séance: $M$.

Francisco IGLESIAS

14h30 Formes politiques républicaines en Europe, XIVe-XVIe siècles

M. Alberto TENENTI, professeur à l'Ecole des Hautes Erudes en Sciences Sociales.

15h La république des Véniticns: genèse d'une î̉ée politique Mone. Elisabeth CROUZET-PAVAN, Docteur d'Etat, chercheur au C.N.R.S.

$15 \mathrm{~h} 30$ Sources de la pensée politique à Lübeck aux XVI et XVII siècles, d'aprés les inventaires de bibliotheques

Mome. Marie-Lonise PELUS, maître de conférence à l' Université de Picardie (Amiens).

16h Pause. Café.

16hl5 Discussion.
MATIN: 9h30 - 12h45

Amphi, Louis Liard

Président de séance: $M$. Carlos

Guilherme MOTA

9 h30 Expériences républicaines en

Angleterre entre 1640 et 1689

M. Jean-Pierre POUSSOU,

professeur à l' Université de

Paris-Sorbonne

(Paris IV)

10h L'idée républicaine dans la révolution américaine $M$. Claude FOHLEN, professeur à l' Université dé
Panthéon-Sorbonne

(Paris I)

10h30 Pause. Café.

$10 \mathrm{~h} 45$ Parlamentarisme et Démocratic dans lé modèle républicain français M. Jean-Marie MAYEUR, professeur à l' Université de Paris-Sorbonne

11 h15 Discussion.

$12 \mathrm{~h} 45$ Déjeuner. 
APR̈ÉS-MIDI: 14h15-18h45

Amphi, Louis Liard

Président de séance: Mome.

Maria Tedda IINHARES

14 hl5 Un président pour les

Etats-Unis: pour quoi faire?

$M$. Andrt KASPI, professeur à

l'Université de

Panthéon-Sorbonne

(Paris I)

14h45 La construction de l' identité nationale pendant la lère

République brésilienne

(1889-1930)

M. Michel DEBRUN, professeur

titulaire à $l^{\prime}$ Université de

Campinas (São Paulo).

$15 \mathrm{~h} 15 \quad$ Etat et classes sociales pendant la lère république brésilienne: une révision historiographique
M. Boris FAUSTO, professeur à l'Université de Săo Paulo.

15h45 Pause. Café.

16h République, travail et citoyenneté

au Bresil, 1889-1945

Mone. Angela Maria de CASTRO

GOMES, professeur à

1'Université Fédérale Fluminense (Rio de Janeiro).

$16 \mathrm{~h} 30$

Réflexions sur

I'industrialisation

brésilienne pendant la périod

républicaine

M. Jacques MAR COVITCH,

Directeur de l'Institut d'Études

Avancées de

l'Université de São Paulo.

17h Discussion.

SAMEDI 20 JANVIER

MATIN: $9 \mathrm{~h}-12 \mathrm{~h} 45$

Amphi, Michelet

Président de séance: $M$. Franfois CROUZET

9h L'idée de République au Brésil $M$. Franciseo IGLESIAS, professeur titulaire à l'Université Fédérale de Minas Gerais.

9h30 Fédération et République Mome. Celia GALVAO QUERINO, professeur à l' Université de São Paulo.

10h Education et citoyenneté pendant la lère république (1889-1930)

Mome. Maria redda LEITE LINHARES, professeur titulaire

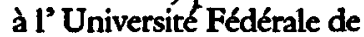
Rio de Janeiro.

10h30 Pause. Café.

10h45 Culture brésilienne ou culture républicaine?

M. Carlos Guilherme MOTA, professeur titulaire à

['Université de Sáo Paulo,

fondateur de

l'Institur d'Études Avancées.
11 hl5 Les militaires: 100 ans de

République

$M$. Jodo QUARTTN DE

MORAES, professeur

à l'Université de

Campinas (São Paulo).

Ilh45 Déjeuner

APRÈS-MIDI: 14h15-18h

Amphi, Michelet

Président de la séance:

Boris FAUSTO

$14 \mathrm{hl5}$ Discussion sur les travaux de la matinée

16h Pause. Caft.

16 h15 Table Ronde

Au-delà de la tradition: quelle république?

Participants:

* M. Le Ministre Luis Carlos BRESSER PEREIRA, professeur à la Fondation Getulio Vargas

* M. Le Governewer Waldir PIRES

* M. João QUARTTM DE MORAES, professeur à $\mathrm{l}^{\prime}$ Université de Campinas (São Paulo)

* M. Femando PACHECO JORDĀO, journaliste. 